\title{
Skin Diseases Detection Using the Spectral Analysis
}

\author{
Xianjiang Meng \\ College of Communication Science and Engineering \\ Shenyang Ligong University \\ Shenyang,China \\ mengxj@jlu.edu.cn \\ Xianli Meng \\ Heilongjiang Branch \\ China Mobile Group Telecommunication Design \\ Institute Co., Ltd \\ Harbin, China \\ kissnoy@sina.com
}

\author{
Jinhua Zhou \\ College of Material Science and Engineering \\ Shenyang Ligong University \\ Shenyang,China \\ zjh06@sohu.com
}

Junjie Wang

College of Physics

Shenyang Ligong University

Shenyang,China

wjjie@sohu.com

\begin{abstract}
In order to detect the skin's disease with the spectral instrument, the reflection spectrum should be gotten to calculate the chromatic values and be analyzed compared. In this paper a kind of chromatic system with self-made fiber probe was designed to measure the spectrum to distinguish the skin normal or not in this paper. The probe was used to minimum the interference outside to the chromatic measurement in order to develop the chromatic measurement accuracy. Through the system, first we got the spectrum of different skin, the normal and the pathological from different part of the sick body, then calculating the tristimulating value and chromatic aberration, we can distinguish the normal with the pathological by the chromatic aberration and make sure if the disease is cured. Good results are achieved by the analyzing of the skin with this system, so a method to distinguish the pathological skin automatically and objectively is attained.
\end{abstract}

Keywords-spectral anaysis;skin disease detection;tristimulating value;fiber probe; chromatic aberration

\section{INTRODUCTION}

The spectrum analysis technology has lots of applications. The main identifying methods are the principal component analysis, the parameter method, the sample irrelevant method and so on. In recent years, the spectrum analysis technology has lots of applications especially in skin diseases [1-3]. It is formal to diagnose the skin diseases through observing of the sick skin for its variation of the color, vein and state. None the less, for so many years people can only get the information with their eyes and experiences to decide the characteristics of the sick skin. In different area, the diseases are different and they cannot be diagnosed with the same method [4-5].

A spectrum analysis and chromatic testing system with self-made fiber probe was devised. According the theory of the chromatics and spectrum analysis and with the optical techniques and computers, still with the medical experiences, an automatic diagnosis system of the skin diseases was established. Through the measuring and analyzing of the general skin diseases, it is proved that the spectrum of the pathological skin over the normal one is different clearly. So the chromatic value is different, and with the different part of the body of the same diseases, the difference of the spectrum is different, so we can decided the type of the skin diseases by the spectrum and decide if the disease is cured by the chromatics [6-8].

\section{THE PRINCIPLE OF ANALYSIS}

The basis of the identifying with the spectrum is the change of their images. The reflected spectrum of the pathological skin over the normal one has changed, the type of the pathological skin is different, and the spectrum diffracted will be different. But the spectrum is difficult to distinguish by peoples' eyes directly, so we change the spectrum into chromatic value, that will be easy to make a distinction.

There are many kinds of skin diseases in medical area [4]. The skin surface is different in color vein and smoothness according different state. Traditionally the doctors can get the first impression of the skin surface with their experiences of many years oneself or maybe experiences from their precursors. The type and status of the skin diseases can be assured. But with this system we use the fiber sensor to get the color image of the pathological skin, comparing the difference of them with the normal; we can get the difference of them after computing.

It is proved that the skin diseases can induce the variation of the two parameters. One is the variation of the reflection spectrum of the pathological skin over the normal, and different diseases induce the variation of the spectrum area; the other is variation of the color. The chromatic aberration can be expressed with the brightness and chromatics [9].

\section{A. The ratio of the relative reflection spectrum}

The skin can be taken as a diffusing reflector. The ratio of the relative reflection spectrum is as followed.

$$
\beta(\lambda)=\alpha(\alpha) \frac{\beta_{s p}(\alpha)}{\beta_{s t}(\alpha)}
$$

Where $\beta_{\text {st }}(\lambda)$ is the reflection spectrum ratio of the standard white plate, $\beta_{\mathrm{sp}}(\lambda)$ is the reflection spectrum ratio 
of the testing skin, $\alpha(\lambda)$ is an adjusting value, it can be decided by the reflection spectrum ratio of the standard white plate with more precious instruments.

We measure the reflection spectrum of the normal skin $\beta_{s 0}(\lambda)$ and of the pathological skin $\beta_{s b}(\lambda)$, the relative reflection spectrum ratio $\beta_{\mathrm{ST}}(\lambda)$ is

$$
\beta_{S T}(\lambda)=\alpha(\lambda) \frac{\beta_{s b}(\lambda)}{\beta_{S D}(\lambda)}
$$

It is obvious that the curve $\beta_{5 \mathrm{r}}(\lambda)$, will be different with the different pathological skin.

\section{B. Chromatic coordinate and chromatic value}

The color of the skin can be expressed by the XYZ chromatic system that is recommended by the Committee of the International Emission (CIE) [10-12]. It uses tristimulating value $\mathrm{XYZ}$ and corresponding chromatic coordinate $\mathrm{x}, \mathrm{y}, \mathrm{z}$ to express the color, and chromatic aberration value $\Delta \mathrm{E}_{\mathrm{ab}}^{*}$

is used to express the difference between two kinds of the color. The color of the skin is the result of the color induced by the invisible emission to the people's eyes, so the color can be expressed by XYZ, and the tri-stimulating value $X Y Z$ can be decided by the stimulated function $\varphi(\lambda)$ is as follows.

$$
\left\{\begin{array}{l}
X=K \int S(\lambda) \beta(\lambda) \bar{x}(\lambda) d \lambda \\
Y=K \int S(\lambda) \beta(\lambda) \bar{y}(\lambda) d \lambda \\
Z=K \int S(\lambda) \beta(\lambda) \bar{z}(\lambda) d \lambda
\end{array}\right.
$$

$\overline{\mathrm{x}}(\lambda), \overline{\mathrm{y}}(\lambda), \bar{z}(\lambda)$ are the tri-stimulating value of the standard observers, The data are form CIE1931; $\Delta \lambda$ is the wavelength interval, here $\Delta \lambda=5 \mathrm{~nm}, \mathrm{~K}$ is the chromatic adjusting factor.

To the skin testing, the chromatic stimulating function $\varphi(\lambda)$ is the function of light source relative spectrum $S(\lambda)$, it is as follows

$$
\varphi(\lambda)=s(\lambda) p(\lambda)
$$

$p(\lambda)$ is the skin reflection spectrum, it comes from formula (1), the light source is bromine-tungsten lamp, so the $S(\lambda)$ is definite.

The CIE chromatic coordinate value $\mathrm{x}, \mathrm{y}, \mathrm{z}$ can get from the tri-stimulating value, the formula is as follows.

$$
\left\{\begin{array}{l}
x=\frac{X}{X+Y+Z} \\
y=\frac{Y}{X+Y+Z} \\
z=\frac{Z}{X+Y+Z}
\end{array}\right.
$$

Additionally, CIE1976 gives a recommended

$\left(L^{*}, a^{*}, b^{*}\right)$ homogeneous chromatic space, it avoids the inhomogeneous chromatic space, the characteristics of the color is expressed with lightness $\mathrm{L}^{*}$ and chromatic values $a^{*}, b^{*}$.

$$
\left\{\begin{array}{c}
\mathrm{L}^{*}=116\left(\frac{Y}{Y_{0}}\right)^{\frac{1}{3}}-16 \\
\mathrm{a}^{*}=500\left[\left(\frac{X}{X_{0}}\right)^{\frac{1}{3}}-\left(\frac{Y}{Y_{0}}\right)^{\frac{1}{3}}\right] \\
\left.\mathrm{b}^{*}=200\left[\left(\frac{Y}{Y_{0}}\right)^{\frac{1}{3}}\right]-\left(\frac{Z}{Z_{0}}\right)^{\frac{1}{3}}\right]
\end{array}\right.
$$

$X_{0}, Y_{0}, Z_{0}$ are the tri-stimulating value of the standard white plate, $X, Y, Z$ are the he tri-stimulating value of the skin, in the system, the chromatic aberration can get from the followed.

$$
\Delta E_{a b}^{*}=\left[\left(\Delta L^{*}\right)^{2}+\left(\Delta a^{*}\right)^{2}+\left(\Delta b^{*}\right)^{2}\right]^{\frac{1}{2}}
$$

$\Delta \mathrm{L}^{*}, \Delta a^{*}, \Delta b^{*}$ are the chromatic aberration of the pathological skin over the normal.

As the boundary of the pathological skin and the normal is blur from the color, so the distinguishing of them can use the fuzzy mathematics method to distinguish in types, only do like this can we get the boundary of the pathological skin. In the experiment we get 50 samples of the skin, measured the spectrum, get the lightness and chromatics.

So we got the mathematical expectation of the skin $\overline{L^{*}}, \overline{a^{*}} \overline{b^{*}}$, mean square deviation $\Delta \mathrm{L}^{*}, \Delta a^{*}, \Delta b^{*}$ and the maximum and minimum of the $\Delta \mathrm{L}^{*}, \Delta a^{*}, \Delta b^{*}$ are $\mathrm{L}_{\mathrm{L}}^{*} \mathrm{~L}_{\mathrm{m}}^{*} \mathrm{a}_{\mathrm{L}}^{*}$ $\mathrm{a}_{\mathrm{n}}^{*} \mathrm{~b}_{\mathrm{L}}^{*} \mathrm{~b}_{\mathrm{n}}^{*}$. They are as followed.

$$
\overline{L^{*}}\left(\overline{a^{*}}, \overline{b^{*}}\right)=\frac{\Sigma_{1}^{N P} L^{*}\left(a^{*} \cdot b^{*}\right)}{\mathbb{B}}
$$

$$
\begin{aligned}
& \Delta \mathrm{L}^{*}\left(\mathrm{a}^{*}, \mathrm{~b}^{*}\right)=\frac{\sqrt{\sum_{\mathrm{i}=1}^{n}\left[\mathrm{~L}_{\mathrm{i}}^{*}\left(a_{\mathrm{i}}^{*}, b_{\mathrm{i}}^{*}\right)-\overline{\left.L^{*}\left(\bar{a}^{*}, \overline{b^{*}}\right)\right]^{2}}\right.}}{N} \\
& \mathrm{~L}_{\mathrm{L}}^{*}\left(a_{\mathrm{L}}^{*}, b_{\mathrm{L}}^{*}\right)=\overline{\mathrm{L}^{*}}\left(\overline{a^{*}}, \overline{b^{*}}\right)-\alpha \Delta \mathrm{L}^{*}\left(\Delta a^{*}, \Delta b^{*}\right) \\
& \mathrm{L}_{\mathrm{n}}^{*}\left(a_{\mathrm{n}}^{*}, b_{\mathrm{n}}^{*}\right)=\overline{\mathrm{L}^{*}}\left(\overline{a^{*}}, \overline{b^{*}}\right)-\alpha \Delta \mathrm{L}^{*}\left(\Delta a^{*}, \Delta b^{*}\right)
\end{aligned}
$$

$\alpha$ is an adjustable parameter, it can vary from $0.5 \sim 1.5$, through adjusting $\alpha$, we can get the upper and lower boundary. The doctors can collect the different samples according the type of the skin diseases. From the samples, the doctors can get the $\alpha$ they want, and get the mathematical expectation and mean square deviation and the boundary is the skin parameters.

Obviously it has a big chromatic aberration $\Delta E_{a b}^{*}$ of the pathological skin over the normal, and with the different pathological skin, the brightness and chromatic value of the skin will vary in the special boundary. 


\section{The structure of the optical fiber probe}

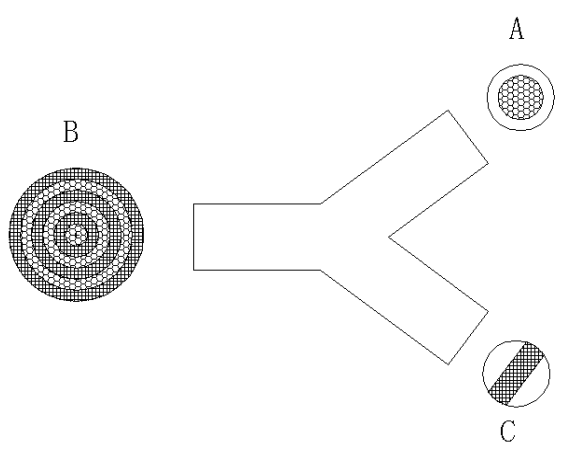

Figure 2. The structure of the optical fiber probe

The structure of the optical fiber probe is shown in Fig 2.The fiber-optic probe consists of three parts: input port A, output part $\mathrm{C}$ and coupled part $\mathrm{B}$. The probe is made of a large variety of stepped multi-mode fiber. The fiber is FG105UCA, wavelength range is $250-1200 \mathrm{~nm}$. The diameter of the core is $105 \pm 2 \mathrm{um}, \mathrm{NA}=0.22 \pm 0.02$. The diameter of port $\mathrm{A}$ and $\mathrm{B}$ is $0.5 \mathrm{~cm}$ and $0.8 \mathrm{~cm}$ respectively, the port $C$ is a $0.3 \mathrm{~cm} * 1.2 \mathrm{~cm}$ rectangular, the leg is $70 \mathrm{~cm}$ in length. The input port $\mathrm{A}$ is a cylindrical shape attached to the light source. The port A is just located at the focus, the light irritates from the light source is reflected by a concave mirror directly into port $\mathrm{A}$ and then into port $\mathrm{B}$. The pot $\mathrm{B}$ is made of the input fiber and output fiber interchangeably. The input light is reflected almost perpendicularly into output fiber and out from port $\mathrm{C}$. The port $\mathrm{C}$ is made into a slit shape in order to match the input port of the monochromator, the port $\mathrm{C}$ is fastened to the slit through a mechanical device to ensure the tight fit, and make sure the most light energy coupled into the monochromator.

\section{THE MEASURING SYSTEM AND OPERATING PRINCIPLE}

The framework chart for the testing system is shown in Fig .1[13]. The light from the light source irradiates into the photomultiplier. In the photomultiplier, the optical grating is driven by a step motor, so the light of different wavelength is diffracted, the output light is converted into electric signal, amplified, $\mathrm{A} / \mathrm{D}$, and put into the computer for manipulating. The final output is the intensity value according the wavelength, and we get the reflection spectrum by normalizing the intensity value. The skin spectrum was gained through the photomultiplier with the self-made optical fiber probe. Every sample at an interval of $5 \mathrm{~nm}$ from $380 \mathrm{~nm}$ to $780 \mathrm{~nm}$, so 81 samples all together.

In the system, a fiber probe is added to decrease the interference of the outside world and focus the light beam in order to increase the sensitivity of the system, and it also can improve the flexibility in measuring.

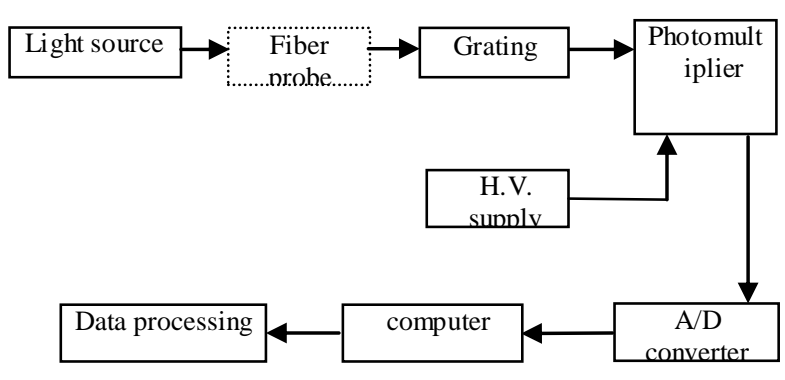

Figure 1. The framework chart of the testing system

\section{EXPRIMENTAL ANALYSIS}

With the system, We tested the spectrum of the different part of the skin, the calculating the chromatic value, analyzing the pathological skin and normal one. In the experiment, the front left arm (normal) and the right cheek (pathological) were tested, the corresponding tristimulating value and chromatic aberration is calculated as followed.

Normal part:

The tri-stimulating value is:

$\mathrm{X}=21.326$

$\mathrm{Y}=28.165$

$\mathrm{Z}=21.048$

The chromatic coordinate :

$\mathrm{x}=0.317$

$\mathrm{y}=0.395$

$\mathrm{z}=0.288$

Pathological part:

The tri-stimulating value is:

$X=9.853$

$\mathrm{Y}=8.762$

$\mathrm{Z}=9.105$

The chromatic coordinate :

$\mathrm{x}=0.367$

$\mathrm{y}=0.338$

$\mathrm{z}=0.295$

Chromatic aberration:

$$
\Delta \mathrm{E}^{*}=28.196
$$

The pathological part was approaching to the normal after curing. The corresponding coordinate and chromatic aberration is as followed.

Normal part:

Normal part 1:

The tri-stimulating value is :

$\mathrm{X}=22.387$

$\mathrm{Y}=24.179$

$\mathrm{Z}=23.145$

The chromatic coordinate :

$\mathrm{x}=0.360$

$\mathrm{y}=0.358$

$\mathrm{z}=0.282$

Normal part 2(The pathological part after treatment) The tri-stimulating value is :

$\mathrm{X}=24.351$ 
$\mathrm{Y}=25.684$

$\mathrm{Z}=23.056$

The chromatic coordinate :

$\mathrm{x}=0.324$

$\mathrm{y}=0.372$

$\mathrm{z}=0.304$

Chromatic aberration:

$$
\Delta \mathrm{E}^{*}=2.162
$$

It is can be seen from the data analysis above, the normal skin and the pathological has the greater chromatic aberration, and after treatment, the chromatic aberration will become smaller, and we also can decide if the skin disease get well by a certain chromatic aberration.

\section{CONCLUSION}

It can be seen from the values that the spectrum of the pathological skin and the normal skin are different, and the tri-stimulating value is different with each other too. So through the medical testing of the different pathological skin, getting the standard spectrum and the chromatics of the samples, forming the skin pathological database, the doctors can make the diagnosis of the skin diseases more objective and quantizing, and further get the information of the extent of the disease treatment.

\section{ACKNOWLEDGEMENT}

This work is supported by the Science and Technology Bureau of Shenyang City of China. Thanks to the cooperation of professor Li Hongda.

\section{REFERENCES}

[1] W. William C. Parson, Modern Optical Spectroscopy, Springer, March 2008

F
[2] A. Fasano ,C. Catassi,Current Approaches to Diagnosis and Treatment of Celiac Disease: An Evolving Spectrum,International Journal of Dermatology,pp. 287-296,April 2010.

[3] P. Park, Sung Yun, J.Kim,The Optimization Variables of Input Data of Artificial Neural Networks for Diagnosing Acute Appendicitis,APPLIED MATHEMATICS \& INFORMATION SCIENCES, Vol.8, pp. 339-343,Jan. 2014

[4] K. Wolff, A. Stuetz,Pimecrolimus for the treatment of inflammatory skin disease, http://informahealthcare.com/doi/abs/10.1517/,Vol.5,pp.643650,Mar. 2011

[5] A. Antonsson, C. Erfurt, K. Hazard,etc. Prevalence and type spectrum of human papillomaviruses in healthy skin samples collected in three continents, The new England journal of medicine, vol. 35 ,pp. 256-264, April 2012.

[6] R. Jamison,E. Mendelsohn, On the chromatic spectrum of acyclic decompositions of graphs,Journal of Graph Theory, Vol 56, pp. 8394,Oct. 2011

[7] G. Angelopoulo, E. Molana,R.Daniilidis, Multispectral skin color modeling, Vol.15,pp. 635-640,Feb. 2013

[8] M. Guillermo; Wang, Lihong; Lin Shao-Pow, Anisotropy in the Absorption and Scattering Spectra of Chicken Breast Tissue, Applied Optics, Vol. 37, pp. 798-804,July 2011

[9] Yang Hongxin,Xie ShuhuiLiHuiLuZukang. Determination of human skin optical properties in vivo from reflectance spectroscopic measurements. Chinese Optics Letters, Vol.5 pp. 181-183,March 2012

[10] B.Tiwari,V.Prakash,V. Tripathi Nonlinear Effects in Optical Fiber Transmission System,IETE TECHNICAL REVIEW,Vol.16,pp. 461-480,May 1999

[11] G. Bobo,Y. Wu, Frosz, Nonlinear fiber-optic strain sensor based on four-wave mixing in microstructured optical fiber,Optics Letters, Vol. 37 pp. 794-796,May 2012

[12] Shokin, I.Yu,O.Shtyrina, Mathematical modelling of nonlinear effects in optical fiber,Russian Journal of Numerical Analysis and Mathematical Modelling,Vol.25,pp. 93-104,Jan. 2010

[13] Meng Xianjiang, et al, Study on the Method to Adjust Optical Fiber's Spectrum Attenuation with the Fourier Transform, Spectroscopy and Spectral Analysis, China,vol.25, pp.544548,April 2009 